\title{
QT dispersion in children with Kawasaki disease
}

\author{
Bo-Kyung Song ${ }^{1}$, Kyoung Sung ${ }^{1}$, Min-Jung Cho ${ }^{2}$, Hyoung-Doo Lee ${ }^{1}$ \\ ${ }^{I}$ Department of Pediatrics, Pusan National University Children's Hospital, Yangsan; \\ ${ }^{2}$ Department of Pediatrics, Pusan National University Hospital, Busan, Korea
}

\begin{abstract}
Background: We analyzed the changes in QT dispersion (QTd) in children with Kawasaki disease (KD), and determined the presence of repolarization abnormality in these children even in the absence of coronary artery abnormalities.

Methods: Ninety-one children with KD and 20 healthy controls were enrolled in this retrospective study. Serial echocardiographic and electrocardiographic (ECG) measurements in the beginning of treatment, 2nd month and 6th month after the diagnosis were compared. Fifty-one of 91 children had at least 2 serial ECG data. The number of patients who had 3 consecutive ECG data was 23.

Results: Among the $67 \mathrm{KD}$ patients with no coronary artery changes, the consecutive mean QTd values were $41.86 \mathrm{~ms}, 37.84 \mathrm{~ms}$, and $25.47 \mathrm{~ms}$, respectively (26 ms for controls). In the analysis of changes among KD patients without coronary artery abnormalities, QTd showed a significant decrease with time $(p=0.01)$. Especially, the 1st month and the 6th month QTd values were significantly different $(p=0.028)$. The mean QTd values in KD patients with coronary artery changes were significantly higher than those in KD patients with no coronary artery changes at each time (1st, 2nd, and 6th month exam).

Conclusion: QTd is significantly increased in children during the early stage of KD. Repolarization abnormality may exist during the acute stage of $K D$, regardless of the echocardiographic changes.
\end{abstract}

Keywords: Kawasaki disease; Electrocardiography

\section{INTRODUCTION}

Changes in coronary arteries such as coronary artery aneurysm and thrombosis are known to be the most prominent cardiac complications of Kawasaki disease (KD) [1]. However, although many patients are believed to have subclinical myocarditis and ventricular dilatation, the clinical significance of coronary artery involvement is not well established [2,3].

QT dispersion (QTd), which is defined as the difference between the longest and shortest QT intervals in a standard 12-lead electrocardiogram (ECG), is thought to reflect a regional difference in the repolarization process [4,5]. The in-

Received: August 13, 2014, Revised: October 16, 2014 ,

Accepted: October 22, 2014

Corresponding Author: Min-Jung Cho, Department of Pediatrics, Pusan National University Hospital, 179 Gudeok-ro, Seo-gu, Busan 602-739, Korea

Tel: +82-51-240-7800, Fax: +82-51-240-6205

E-mail: mjchomd@gmail.com crease in QTd has been reported in many forms of heart disease such as dilated cardiomyopathy, hypertrophic cardiomyopathy, and long QT syndrome [6-9]. The investigators suggested that an increase in QTd reflects electrophysiological instability of the myocardium; therefore, it is associated with an increased risk of ventricular tachyarrhythmia and sudden cardiac death under various pathological conditions [9-12].

To determine the changes in QTd in children with $\mathrm{KD}$ and to determine the presence of repolarization abnormality in these children even in the absence of coronary artery abnormalities, we examined the ECGs in a cohort of children with $\mathrm{KD}$ and healthy controls.

\section{MATERIALS AND METHODS}

We performed a retrospective analysis of data obtained in all of the patients with $\mathrm{KD}$, who were diagnosed and treated at our institute from January 2008 to July 2012. The medical 
records of all patients were reviewed to obtain data regarding demographics, clinical features leading diagnosis, and management of KD. All of the patients had fulfilled the diagnostic criteria of $\mathrm{KD}$ and had received intravenous immunoglobulins $(2 \mathrm{~g} / \mathrm{kg})$ and high-dose oral aspirin $(50 \mathrm{mg} / \mathrm{kg} /$ day $)$ as the primary therapy for $\mathrm{KD}$.

Serial echocardiography and ECG were performed in each subject at the time of diagnosis, 2nd month, and 6th month after the diagnosis, which represent acute, subacute, chronic phase of KD respectively. The 12-lead ECG was recorded at $25 \mathrm{~mm} / \mathrm{sec}$ paper speed. QT intervals were measured manually in all of the 12 leads by 2 blinded investigators who unaware of echocardiographic findings. The QT interval was measured from the onset of the QRS complex to the end of the $\mathrm{T}$ wave at the level of the TP isoelectric baseline. If $\mathrm{U}$ waves were present, the end of $\mathrm{T}$ wave was taken as the nadir between $\mathrm{T}$ and $\mathrm{U}$ waves. When it was difficult to define the $\mathrm{T}$ wave offset, it was measured at the intersection of the isoelectric baseline and the maximum tangent line of the $\mathrm{T}$ wave terminal limb. A main investigator and a second investigator measured QT intervals independently. When the values from them showed significantly different intervals, they rechecked whether the measurement was done by the above-mentioned method. When the end of the $\mathrm{T}$ wave could not be defined using the method, the lead was excluded; of the 1980 leads obtained (12-36 per subject), 84 leads were excluded from the analysis.

Echocardiographic data and measurements of coronary artery diameters were obtained from the final reports. The degree of coronary artery involvement was classified according to the Japanese Ministry of Health Criteria [13]. Mild coronary artery disease $(\mathrm{CAD})$ was defined as the coronary artery internal diameter of 3 to $8 \mathrm{~mm}$ at less than 5 years of age or 4 to $8 \mathrm{~mm}$ at 5 years of age or older without obstruction or thrombosis of coronary arteries. The internal diameter larger than $8 \mathrm{~mm}$ was defined as giant aneurysm.

A total of $91 \mathrm{KD}$ patients and 20 healthy control patients were included in this analysis. The control group was randomly selected from the clinical pediatric population who visited our clinic for nonspecific chest pain. Chest pain of the controls was confirmed as noncardiac origin by physical examination, ECG, and echocardiography. Subjects were excluded if they had pre-existing arrhythmia, congenital heart disease or cardiopulmonary problem.
This study was exempt from approval of the institutional review board (IRB) because retrospective chart review collected clinical records without personal information. And every process of the protocol was done within the routine management of $\mathrm{KD}$ without additional examination or invasive procedure for this study. The data of control group was also collected through routine work-up of chest pain.

Results were presented as mean \pm standard deviation. Comparison of continuous variables between groups was performed using one-way analysis of variance (ANOVA). All analyses were performed using IBM SPSS version 19.0 (IBM Co., Armonk, NY, USA). For all tests, $p<0.05$ was considered statistically significant.

\section{RESULTS}

\section{Demographics of the Patients}

Age and sex distribution among $91 \mathrm{KD}$ patients is described in Table 1. There were no significant differences in gender and age distribution between patients and control group (data not shown). Among these 91 patients, more than 2 serial ECG data could be collected in 51 patients. The number of patients who had all three consecutive ECG data was 23 (Fig. 1A). Of these 91 patients, 23 patients showed echocardiographic coronary artery lesions; especially in 6 patients, a giant aneurysm was identified. The lesions were not normalized in $7 \mathrm{pa}-$ tients after a follow up of 6 months (Fig. 1B).

\section{QT Dispersion in Patients without Coronary Lesions during the Complete Follow-up Period}

In 68 patients, there were no coronary artery changes from the time of diagnosis. There were no age- or sex-related differences in QTd in these patients. Of these 68 patients, we were able to obtain three consecutive follow-up ECGs in 12 cases;

Table 1. Age and sex distribution of patients $(n=91)$

\begin{tabular}{llc}
\hline Characteristic & & Value \\
\hline \multirow{2}{*}{ Gender } & Male & $55(60.4)$ \\
& Female & $36(39.6)$ \\
\multirow{2}{*}{ Age at diagnosis (yr) } & Mean \pm SD & $2.84 \pm 2.04$ \\
& Range & $0.17-10$ \\
& Median & 2.46 \\
\hline
\end{tabular}

Values are presented as mean \pm SD or number (\%). $\mathrm{SD}$, standard deviation. 

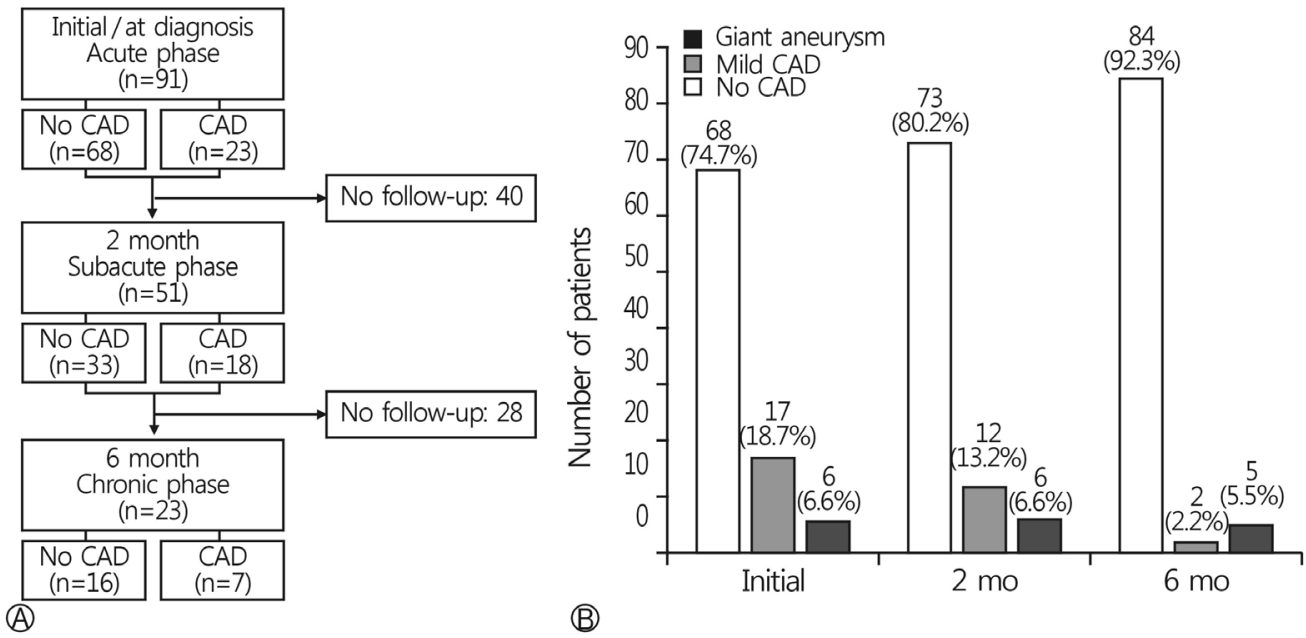

Fig. 1. (A) Flow chart of electrocardiogram follow-up during the study. (B) Change in echocardiographic findings of patients during 6 months from the diagnosis of Kawasaki disease. CAD, coronary artery disease.

the mean QTd was $41.86 \pm 2.12 \mathrm{msec}$ in the initial month, $37.84 \pm 1.40 \mathrm{msec}$ in the 2 nd month after the diagnosis, and $25.47 \pm 1.81 \mathrm{msec}$ in the 6 th month after the diagnosis. The initial QTd value did not show a statistically significant difference compared to the QTd value at the1st follow-up; however, the mean initial QTd was significantly increased as compared to either the value at the last follow-up $(p=0.00)$ or the value in the control group (mean QTd in the control group, 26.00 $\pm 2.12 \mathrm{msec} ; p=0.00$ ) (Fig. 2). The mean QTd value at the 6 th month after diagnosis was not significantly different from the mean QTd value in the control group.

\section{QT Dispersion in Patients with Coronary Lesions during the Complete Follow-up Period}

On comparison of the initial QTd between patients with coronary lesions and patients without coronary lesions, QTd was significantly greater in patients with coronary lesions $(50.82 \pm 3.12 \mathrm{msec}$; median $52 \mathrm{msec})$ than in those without coronary lesions $(39.89 \pm 2.18 \mathrm{msec}$; median $40.00 \mathrm{msec})$ $(p=0.011)$ (Fig. 3A).

At 2 months after the diagnosis, the comparison of QTd between patients without coronary lesions and those with coronary lesions also showed a significant difference; $37.79 \pm$ $1.32 \mathrm{msec}$ with a median of $40.00 \mathrm{msec}$ for the patients without coronary lesions vs. $48.85 \pm 3.08 \mathrm{msec}$ with a median of $52.00 \mathrm{msec}$ for the patients with coronary lesions $(p=0.002)$ (Fig. 3B).

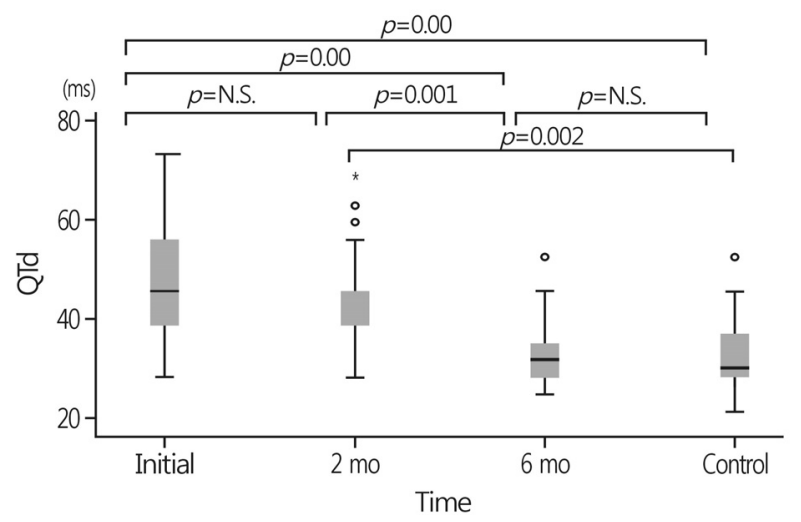

Fig. 2. Comparison of QTd at different times among patients with Kawasaki disease who never had coronary artery disease. QTd, QT dispersion; SD, standard deviation.

Similarly, the difference in the mean QTd between patients without coronary lesions $(27.25 \pm 1.77 \mathrm{msec}$; median $24 \mathrm{msec})$ and those with coronary lesions $(53.55 \pm 6.97 \mathrm{msec} ; 54.00$ msec) at the 6-month follow-up was also significant $(p=0.001)$ (Fig. 3C).

\section{DISCUSSION}

$\mathrm{KD}$ is a systemic vasculitis affecting medium and small sized blood vessels, particularly the coronary arteries. Within the spectrum of the inflammatory process, children can present with hemodynamic abnormalities secondary to myocarditis or pericarditis [1]. Accordingly, ventricular dysfunction and arrhythmias may occur, and may do so independent of coro- 

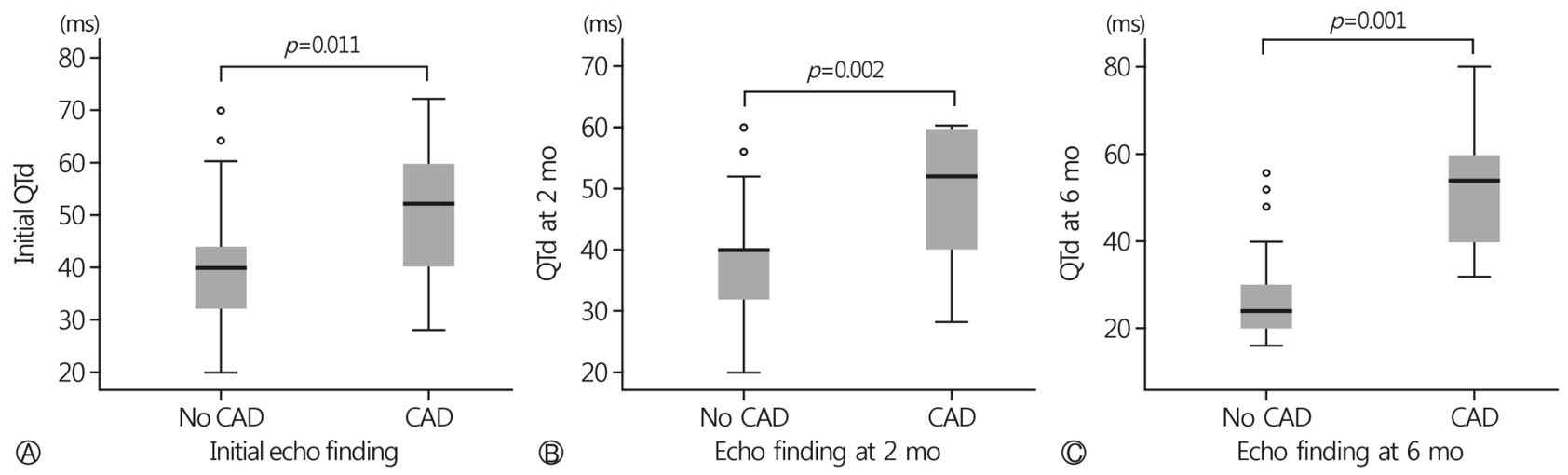

Fig. 3. (A) Comparison of QTd in patients at the initial diagnosis of Kawasaki disease. (B) Comparison of QTd in patients at 2 months after the diagnosis of Kawasaki disease (subacute phase). (C) Comparison of QTd in patients at the 6-month follow-up (chronic phase). QTd, QT dispersion; CAD, coronary artery disease.

nary artery involvement [3]. It has been postulated that such an involvement of the myocardium and coronary arteries may result in unrecognized ischemia in the ventricular myocardium causing electrophysiological abnormalities in ventricular forces and repolarization $[2,14-16]$. The relationship between the natural course of $\mathrm{KD}$ and these electrical abnormalities has not been well established.

QTd is thought to reflect spatial heterogeneity of ventricular refractoriness [5]. Therefore, it has been speculated that increased QTd is a sign of regional differences in the duration of ventricular repolarization. It has been assessed in a variety of disorders. Abnormal QTd was identified in adults with hypertrophic cardiomyopathy and myocardial infarction $[6-8,10]$. Among children, such an abnormality was documented in those with dilated cardiomyopathy and ventricular arrhythmia [12]. The speculated threshold value of QTd for identifying the high risk group was $90 \mathrm{msec}$; an increase in QTd above this value may be an important risk factor for arrhythmias and sudden cardiac death $[10,17,18]$.

There have been several studies assessing QTd in patients with KD. Results of previous studies mainly suggested that increased QTd positively correlated with the severity of coronary changes in patients with $\mathrm{KD}[14,15]$. These results were in agreement with those of our study with respect to identifying significantly increased QTd in patients with coronary changes.

In the present study, we showed that the degree of QTd was significantly increased in the patients in the acute phase of $\mathrm{KD}$ compared with those in the recovery phase of $\mathrm{KD}$, regardless of the coronary artery pathology. This result may indicate that the inflammatory process caused by $\mathrm{KD}$ may affect the myocardium and the coronary microcirculation, leading to heterogeneity of ventricular repolarization. It seems that our results are conflict with previous results: Osada et al. reported significantly longer QTd in $\mathrm{KD}$ patients with giant aneurysm, and Dahdah et al. presented that QTd was significantly greater in patients with persistent coronary aneurysm than in those with early resolution of aneurysm $[14,15]$. However, methods of these studies do not correspond with ours. These studies obtained ECG data after several years of the onset of illness. ECG data during acute phase was not included in these studies. It may be worth making further studies about electophysiologic change in KD patients that cover both of acute phase and long term follow-up.

The major limitation of this study is that it was a retrospective analysis. The number of patients was probably small because large numbers of KD patients dropped out from follow-up course of 6 month-follow-up, especially patients without CAD.

In conclusion, we note significant heterogeneity of ventricular repolarization in patients in the acute stage of $\mathrm{KD}$, irrespective of the presence of coronary sequelae at the onset of $\mathrm{KD}$. This may represent risk for developing arrhythmia during this stage.

\section{REFERENCES}

1. McCrindle BW. Kawasaki disease: a childhood disease with important consequences into adulthood. Circulation 2009; 120:6-8. 
2. Crystal MA, Syan SK, Yeung RS, Dipchand AI, McCrindle BW. Echocardiographic and electrocardiographic trends in children with acute Kawasaki disease. Can J Cardiol 2008;24: 776-80.

3. Ghelani SJ, Singh S, Manojkumar R. QT interval dispersion in North Indian children with Kawasaki disease without overt coronary artery abnormalities. Rheumatol Int 2011;31:301-5.

4. Malik M, Batchvarov VN. Measurement, interpretation and clinical potential of QT dispersion. J Am Coll Cardiol 2000; 36:1749-66.

5. Day CP, McComb JM, Campbell RW. QT dispersion: an indication of arrhythmia risk in patients with long QT intervals. Br Heart J 1990;63:342-4.

6. Ueda H, Hayashi T, Tsumura K, Kaitani K, Yoshimaru K, Nakayama $\mathrm{Y}$, et al. QT dispersion and prognosis after coronary stent placement in acute myocardial infarction. Clin Cardiol 2007;30:229-33.

7. Hashimoto N, Musha H, Ozawa A, Imai Y, Kawasaki K, Miyazu O, et al. Relationship between infarction location and size to QT dispersion in patients with chronic myocardial infarction. Jpn Heart J 2002;43:455-61.

8. Bayrak F, Kahveci G, Mutlu B, Değertekin M, Demirtaş E. Usefulness of surface electrocardiogram in predicting the clinical course of patients with hypertrophic cardiomyopathy. Anadolu Kardiyol Derg 2007;7(Suppl 1):178-81.

9. Priori SG, Napolitano C, Diehl L, Schwartz PJ. Dispersion of the QT interval.a marker of therapeutic efficacy in the idiopathic long QT syndrome. Circulation 1994;89:1681-9.

10. Buja G, Miorelli M, Turrini P, Melacini P, Nava A. Comparison of QT dispersion in hypertrophic cardiomyopathy between patients with and without ventricular arrhythmias and sudden death. Am J Cardiol 1993;72:973-6.

11. Fu GS, Meissner A, Simon R. Repolarization dispersion and sudden cardiac death in patients with impaired left ventricular function. Eur Heart J 1997;18:281-9.

12. Dubin AM, Rosenthal DN, Chin C, Bernstein D. QT dispersion predicts ventricular arrhythmia in pediatric cardiomyopathy patients referred for heart transplantation. J Heart Lung Transplant 1999;18:781-5.

13. Research Committee on Kawasaki Disease. Report of subcommittee on standardization of diagnostic criteria and reporting of coronary artery lesions in Kawasaki disease. Tokyo: Japanese Ministry of Health and Welfare; 1984.

14. Osada M, Tanaka Y, Komai T, Maeda Y, Kitano M, Komori $\mathrm{S}$, et al. Coronary arterial involvement and QT dispersion in Kawasaki disease. Am J Cardiol 1999;84:466-8.

15. Dahdah NS, Jaeggi E, Fournier A. Electrocardiographic depolarization and repolarization: long-term after Kawasaki disease. Pediatr Cardiol 2002;23:513-7.

16. Kuriki M, Fujino M, Tanaka K, Horio K, Kusuki H, Hosoi $\mathrm{M}$, et al. Ventricular repolarization lability in children with Kawasaki disease. Pediatr Cardiol 2011;32:487-91.

17. Bogun F, Chan KK, Harvey M, Goyal R, Castellani M, Niebauer M, et al. QT dispersion in nonsustained ventricular tachycardia and coronary artery disease. Am J Cardiol 1996; 77:256-9.

18. Anastasiou-Nana MI, Nanas JN, Karagounis LA, Tsagalou EP, Alexopoulos GE, Toumanidis S, et al. Relation of dispersion of QRS and QT in patients with advanced congestive heart failure to cardiac and sudden death mortality. Am J Cardiol 2000;85:1212-7. 